\title{
Asymptotic Boundary Conditions and Numerical Methods for Nonlinear Elliptic Problems on Unbounded Domains*
}

\author{
By T. M. Hagstrom and H. B. Keller
}

\begin{abstract}
We present a derivation and implementation of asymptotic boundary conditions to be imposed on "artificial" boundaries for nonlinear elliptic boundary value problems on semi-infinite "cylindrical" domains. A general theory developed by the authors in [11] is applied to establish the existence of exact boundary conditions and then to obtain useful approximations to them. The derivation is based on the Laplace transform solution of the linearized problem at infinity. We discuss the incorporation of the asymptotic boundary conditions into a finite-difference scheme and present the results of numerical experiments on the solution of the Bratu problem in a two-dimensional stepped channel. We also touch on certain problems concerning the existence of solutions of this problem on infinite domains and conjecture on the behavior of the critical parameter value with respect to changes in the domain. Some numerical evidence supporting the conjecture is given.
\end{abstract}

1. Introduction. There are basically only two methods by means of which problems posed on infinite domains can be "replaced" by problems posed on finite domains. The first rather obvious method is to map the infinite domain into a finite domain with as smooth a mapping as possible. But of course the mapping cannot be bounded and so the resulting mapped problem must contain singular points in its finite domain. To treat this new problem numerically one must then face the nasty problem of approximations about a singularity. This important area of approximation theory is poorly developed and consequently this approach is seldom used in practice. For nonlinear problems and problems posed in two or more dimensions the difficulties are indeed formidable.

The second method of reduction to a finite domain is to introduce an artificial finite boundary and to consider the problem only on the enclosed finite domain. Of course one must then impose some "appropriate" boundary conditions on the artificial boundary. What "appropriate" means is not always discussed properly. Indeed a host of work is done devising "nonreflecting" or "soft" or "absorbing" boundary conditions without relating the new reduced problem to the original problem. Frequently, this is not even noticed as the problem on the infinite domain

Received May 17, 1985; revised March 31, 1986.

1980 Mathematics Subject Classification (1985 Revision). Primary 35J25, 65N99.

Key words and phrases. Asymptotic boundary conditions, asymptotic expansions, artificial boundaries, unbounded domains.

* Supported by the U.S. Army Research Office under Contract No. DAAG29-85-K-0092 and by the U.S. Department of Energy under Contract No. DE-AM03-76SF-00767. 
may not have been completely formulated (i.e., a well-posed problem is not always presented). These attempts to use artificial boundaries without relating the approximation to a well-posed problem essentially seek to avoid the difficulties inherent in the previous mapping method by ignoring the singularities.

However, it is possible to use the introduction of artificial boundaries in a completely rigorous and practical way for many problems. It has been done by several authors and we briefly point out the most important such contributions at the end of this section. Our approach to this technique is to seek to replace the original well-posed problem for the infinite domain by an "equivalent" well-posed problem on a finite domain. By equivalent we mean that the solutions to the two problems (finite and infinite) agree on the finite domain that is common to the two formulations. While it may be possible to do this in principle, the required auxiliary data, such as projection operators and special solutions in a neighborhood of infinity, are not generally available. Thus they must be approximated, and in doing so we obtain a sequence of asymptotic boundary conditions, of successively higher orders, for the finite domain problem. This theory has been developed for quite general partial differential equations by the authors in [11], [12]. It is a rather natural extension of related work on ordinary differential equations over infinite intervals by Keller [15], Lentini [19], Jepson [13], Jepson and Keller [14] and Keller and Lentini [17].

In this paper we show how our theory applies to nonlinear elliptic problems in infinite "cylindrical" domains. Specifically, we consider semilinear elliptic equations of the form

$$
\text { (a) } L u(x, y) \equiv \frac{\partial^{2} u}{\partial x^{2}}+L_{1} \frac{\partial u}{\partial x}+L_{2} u=f(u, \mathbf{y}) \text {, }
$$

posed on unbounded cylindrical domains in $\mathbf{R}^{N+1}$ :

$$
(x, \mathbf{y}) \in[0, \infty) \times \Omega, \quad \Omega \subseteq \mathbf{R}^{N} .
$$

On the lateral surface of this domain we pose conditions independent of $x$, say,

$$
\text { (b) } a_{\partial \Omega}(\mathbf{y}) \frac{\partial u}{\partial \nu}+b_{\partial \Omega}(\mathbf{y}) u=c_{\partial \Omega}(\mathbf{y}) ; \quad x \in[0, \infty), \mathbf{y} \in \partial \Omega
$$

At the base of the cylinder we require

$$
\text { (c) } b_{1}(\mathbf{y}) \frac{\partial u}{\partial x}+b_{0}(\mathbf{y}) u=c_{0}(\mathbf{y}), \quad x=0, \mathbf{y} \in \Omega \text {. }
$$

As $x \rightarrow \infty$ the conditions are that

$$
\text { (i) } \lim _{x \rightarrow \infty} u(x, \mathbf{y})=u_{\infty}(\mathbf{y})
$$

(ii) $\lim _{x \rightarrow \infty} \frac{\partial u(x, \mathbf{y})}{\partial x}=0$.

The operator $L$ in (1.1(a)) is uniformly elliptic, second-order, with coefficients independent of $x$, say,

$$
\begin{aligned}
& \text { (a) } L_{1} \equiv \sum_{j=1}^{N} a_{0 j}(\mathbf{y}) \frac{\partial}{\partial y_{j}}+a_{0}(\mathbf{y}) \\
& \text { (b) } L_{2} \equiv \sum_{\substack{i=1 \\
j=1}}^{N} \frac{\partial}{\partial y_{i}}\left(a_{i j}(\mathbf{y}) \frac{\partial}{\partial y_{j}}\right)+a(\mathbf{y}), \quad a_{i j} \equiv a_{j i} .
\end{aligned}
$$


In (1.1(b)) the conormal derivative associated with $L$ on $\partial \Omega$ is denoted by $\partial / \partial \nu$. Specifically, if $\boldsymbol{v}(\mathbf{y}) \equiv\left(\nu_{1}, \ldots, \nu_{N}\right)$ is the unit outward normal to $\partial \Omega$, then using (1.1(a)) and (1.2),

$$
\begin{aligned}
& \text { (c) } \frac{\partial}{\partial \nu} \equiv \frac{\partial}{\partial \nu_{\perp}}+\frac{1}{2} \sum_{i=1}^{N} \nu_{i}(y) a_{0 i}(\mathbf{y}) \frac{\partial}{\partial x}, \quad a_{i 0} \equiv a_{0 i} \\
& \text { (d) } \frac{\partial}{\partial \nu_{\perp}} \equiv \sum_{\substack{i=1 \\
j=1}}^{N} \nu_{i}(\mathbf{y}) a_{i j}(\mathbf{y}) \frac{\partial}{\partial y_{j}}
\end{aligned}
$$

The conditions $(1.1(\mathrm{~d}))$ as $x \rightarrow \infty$ are that $u(x, y)$ should approach the solution of the limiting problem in the cross section:

$$
\begin{array}{ll}
\text { (a) } L_{2} u_{\infty}(\mathbf{y})=f\left(u_{\infty}(\mathbf{y}), \mathbf{y}\right), & \mathbf{y} \in \Omega \\
\text { (b) } a_{\partial \Omega}(\mathbf{y}) \frac{\partial u_{\infty}}{\partial \nu_{\perp}}+b_{\partial \Omega}(\mathbf{y}) u_{\infty}=c_{\partial \Omega}(\mathbf{y}), & \mathbf{y} \in \partial \Omega
\end{array}
$$

Problems of the above form arise in many applications. Semilinear elliptic equations are common in chemical reaction-diffusion processes (see Aris [3]), joule heating (see Keller and Cohen [16]), etc. The methods we describe can be applied to much more general systems, to higher-order equations, and we can allow variations with $x$ in both the domain shape and in the coefficients. In the latter case, some asymptotic conditions need only be imposed as $x \rightarrow \infty$, to approach the above conditions, and our theory remains valid. These extensions are discussed in Hagstrom [12] and Hagstrom and Keller [11]. Indeed, the problem we study in Section 4 has a noncylindrical domain.

To treat problem (1.1) numerically, we introduce an artificial boundary at some location $x=\tau>0$ and seek to impose appropriate boundary conditions there over $\Omega$. Then we need only solve (numerically) a more or less standard boundary value problem over $[0, \tau] \times \Omega$. A theory to characterize exact boundary conditions over such artificial boundaries has been developed by the authors in [11], [12]. In most applications, these exact solutions can only be approximated. One of the main purposes of this work is to illustrate the power of this general theory and to demonstrate the use of the approximate asymptotic boundary conditions on a nonlinear problem.

Other authors have discussed boundary conditions at artificial boundaries for linear elliptic problems. Gustafsson and Kreiss [10] point out the possibility of deriving exact conditions by means of Laplace transforms in $x$. Fix and Marin [7] and Goldstein [9] use a related approach to treat problems in underwater acoustics and wave propagation in cylindrical waveguides. The first approximation in our asymptotic boundary conditions agrees with what would be obtained by applying the Laplace transform method to the linearized problem about $u_{\infty}(\mathbf{y})$.

In Section 2 we summarize the basic results of [11] and show how they apply to problem (1.1). In particular, we derive the asymptotic boundary conditions (ABC) and state conditions for their validity. In Section 3 we show one way to employ the $\mathrm{ABC}$ in a discretization and discuss their efficient implementation. Finally in Section 4 the Bratu problem in a two-dimensional stepped channel is considered. Some 
theoretical results for finite domains are recalled and used. Numerical results are presented to show the effects of varying the location of the artificial boundary and of varying the order of approximation in the $A B C$. Some questions and numerical experiments relating to the existence of solutions in unbounded domains are also examined.

2. The Asymptotic Boundary Conditions: "ABC". We assume the existence of a solution, $u_{\infty}(\mathbf{y})$, of the limiting problem (1.3). Then, with the definition

$$
v(x, \mathbf{y}) \equiv u(x, \mathbf{y})-u_{\infty}(\mathbf{y}),
$$

we write (1.1(a)) as

$$
\begin{aligned}
& \text { (a) } \mathscr{L}_{v} \equiv L v-f_{u}\left(u_{\infty}(\mathbf{y})\right) v=r(v) \\
& \text { (b) } r(v) \equiv f\left(u_{\infty}+v\right)-f_{u}\left(u_{\infty}\right) v-f\left(u_{\infty}\right) .
\end{aligned}
$$

Note that $r(v)=\mathcal{O}\left(|v|^{2}\right)$ as $|v| \rightarrow 0$. Since the lateral boundary conditions (1.1(b)) are independent of $x$ and $u_{\infty}(\mathbf{y})$ satisfies them, we have for $v(x, y)$ the homogeneous conditions

$$
\text { (c) } a_{\partial \Omega}(\mathbf{y}) \frac{\partial v}{\partial \nu}+b_{\partial \Omega}(\mathbf{y}) v=0, \quad x \in[0, \infty), \mathbf{y} \in \partial \Omega .
$$

The conditions, (1.1(d)), as $x \rightarrow \infty$ now become, in terms of $v$,

$$
\text { (i) } \lim _{x \rightarrow \infty} v(x, \mathbf{y})=0 \text {, }
$$

(ii) $\lim _{x \rightarrow \infty} \frac{\partial v}{\partial x}(x, \mathbf{y})=0$.

For the present, we ignore the conditions (1.1(c)) on the base of the cylinder, $x=0$, and seek solutions of (2.2) in the "tail": $x>\tau$ for some $\tau>0$. After all, if problem (1.1) has a solution then (2.2) must have a solution in the tail. Our goal is to find conditions at $x=\tau$ that the solution in the tail must satisfy.

In [11] we establish sufficient conditions for problems of the form (2.2) to have solutions in the tail. To apply this theory here, and to use the same notation, we reformulate the problem as an ordinary differential equation in a Banach space. Thus we introduce the two component vectors

$$
\text { (a) } \mathbf{w}(x) \equiv\left(\begin{array}{c}
w_{1}(x, \mathbf{y}) \\
w_{2}(x, \mathbf{y})
\end{array}\right) \equiv\left(\begin{array}{c}
v(x, \mathbf{y}) \\
\frac{\partial v}{\partial x}(x, \mathbf{y})
\end{array}\right),
$$

and for each $x \geqslant \tau$ they are to lie in a Banach space $\mathscr{B}$ defined by

$$
\text { (b) } \mathscr{B} \equiv\left\{\mathbf{w}: w_{1} \in W_{2}^{0}(\Omega), w_{2} \in W_{1}(\Omega)\right\} .
$$

Here, $W_{1}(\Omega)$ and $W_{2}(\Omega)$ are Sobolev spaces and

$$
\text { (c) } W_{2}^{0}(\Omega) \equiv\left\{v: v(\mathbf{y}) \in W_{2}(\Omega), a_{\partial \Omega}(\mathbf{y}) \frac{\partial v}{\partial \nu}+b_{\partial \Omega}(\mathbf{y}) v=0, \mathbf{y} \in \partial \Omega\right\} .
$$

Thus elements of $W_{2}^{0}(\Omega)$ satisfy the homogeneous boundary conditions $(2.2(\mathrm{c}))$. 
We introduce the linear and nonlinear operators $A$ and $R$ as
(a) $A \mathbf{w} \equiv\left(\begin{array}{cc}0 & I \\ -L_{2}+f_{u}\left(u_{\infty}(y)\right) & -L_{1}\end{array}\right) \mathbf{w}=\left(\begin{array}{c}w_{2} \\ -L_{2} w_{1}+f_{u}\left(u_{\infty}\right) w_{1}-L_{1} w_{2}\end{array}\right)$,
(b) $\quad R(\mathbf{w}) \equiv\left(\begin{array}{c}0 \\ r\left(w_{1}\right)\end{array}\right) \equiv\left(\begin{array}{c}0 \\ f\left(u_{\infty}+w_{1}\right)-f\left(u_{\infty}\right)-f_{u}\left(u_{\infty}\right) w_{1}\end{array}\right)$.

Now (2.2) can be formulated in the tail as

$$
\begin{aligned}
& \text { (a) } \frac{d \mathbf{w}}{d x}-A \mathbf{w}=R(\mathbf{w}), \quad x>\tau, \mathbf{w}(x) \in \mathscr{B} ; \\
& \text { (b) } \lim _{x \rightarrow \infty} \mathbf{w}(x)=\mathbf{0} .
\end{aligned}
$$

To solve (2.5), we must first study initial value or Cauchy problems for the linear operator on the left-hand side of $(2.5(\mathrm{a}))$. Thus we consider

$$
\begin{aligned}
& \text { (a) } \frac{d \mathbf{w}}{d x}-A \mathbf{w}=\mathbf{0}, \quad x>\tau, \mathbf{w} \in \mathscr{B} ; \\
& \text { (b) } \mathbf{w}(\tau)=\xi \in \mathscr{B} .
\end{aligned}
$$

The solution of (2.6) is formally represented in terms of the solution operator $S\left(x, x^{\prime}\right)$ as

$$
\mathbf{w}(x)=S(x, \tau) \xi .
$$

In particular, we are interested in all initial data for which the solution satisfies (2.5(b)). Since the equation and the condition at $\infty$ are homogeneous, this data must form a subspace of $\mathscr{B}$-we call it the admissible subspace:

$$
\mathscr{A}(\tau) \equiv\left\{\boldsymbol{\eta}: \boldsymbol{\eta} \in \mathscr{B}, \lim _{x \rightarrow \infty} S(x, \tau) \eta=\mathbf{0}\right\} .
$$

In general, we can characterize $\mathscr{A}(\tau)$ by means of the spectrum $\left\{\lambda_{k}\right\}$ of the operator $A$ and its eigenfunctions, $\left\{\phi_{k}\right\}$, that is,

$$
\begin{aligned}
& \text { (a) } A \phi_{k}=\lambda_{k} \phi_{k}, \quad k=1,2, \ldots,\left\|\phi_{k}\right\| \neq 0 \\
& \text { (b) } \phi_{k}(\mathbf{y}) \in \mathscr{B} .
\end{aligned}
$$

Recall, from (2.3(b), (c)), that boundary conditions are imposed by means of (2.9(b)). Using (2.4(a)) and the above we see that this eigenvalue problem is equivalent to the scalar elliptic eigenvalue problem

$$
\begin{aligned}
& \text { (a) } L_{2} \phi_{k}-f_{u}\left(u_{\infty}(\mathbf{y})\right) \phi_{k}+\lambda_{k} L_{1} \phi_{k}+\lambda_{k}^{2} \phi_{k}=0, \quad \mathbf{y} \in \Omega ; \\
& \text { (b) } a_{\partial \Omega}(\mathbf{y}) \frac{\partial \phi_{k}}{\partial \nu}+\frac{\lambda_{k}}{2} \sum_{i=1}^{N} \nu_{i}(\mathbf{y}) a_{0 i}(\mathbf{y}) \phi_{k}+b_{\partial \Omega}(\mathbf{y}) \phi_{k}=0, \quad \mathbf{y} \in \partial \Omega .
\end{aligned}
$$

Then we have

$$
\text { (c) } \phi_{k}(\mathbf{y})=\left(\begin{array}{c}
\phi_{k}(\mathbf{y}) \\
\lambda_{k} \phi_{k}(\mathbf{y})
\end{array}\right) \equiv\left(\begin{array}{c}
1 \\
\lambda_{k}
\end{array}\right) \phi_{k}(\mathbf{y})
$$


Solutions of (2.6) can be represented in terms of these eigenfunctions by simple separation of variables or Laplace transforms, provided

(a) The eigenvalues, $\lambda_{k}$, are distinct and bounded away from the imaginary axis;

(b) The eigenfunctions, $\phi_{k}$, form a Riesz basis** for $\mathscr{B}$.

We assume that (2.11) holds for the remainder of our study. In the terminology of [11], conditions (2.11(a)) insure that the linearized problem has an exponential dichotomy. This seems necessary for the nonlinear problems which we treat here. It assures that $u_{\infty}(y)$ is "spatially stable," say as $x \rightarrow \infty$, and without this, it would be difficult to satisfy $(1.1(\mathrm{~d}))$. We do not require exponential dichotomies for linear problems, but make do with ordinary dichotomies; see [11] for more details. The completeness in (2.11(b)) can be difficult to check for nonselfadjoint problems; the selfadjoint case is discussed in Berezanskii [6] and Agmon [1], who treats the case with $L_{1} \equiv 0$. In Agmon and Nirenberg [2] sufficient conditions are given for completeness in the class of initial data yielding absolutely integrable solutions in the tail.

Formally, solutions of (2.6) are given in terms of the eigenfunctions of (2.9) by expansions of the form

$$
\text { (a) } \mathbf{w}(x, \xi)=\sum_{k} c_{k}(\xi) \phi_{k}(\mathbf{y}) e^{\lambda_{k}(x-\tau)},
$$

where of course, for any $\xi \in \mathscr{B}$,

$$
\text { (b) } \xi=\sum_{k} c_{k}(\xi) \phi_{k}(\mathbf{y}) \text {. }
$$

The expansion coefficients $c_{k}(\xi)$ are given in terms of the eigenfunctions $\psi_{k}(\mathbf{y})$ of the adjoint problem to (2.9) or (2.10) by an appropriate inner product, say,

(c) $c_{k}(\xi) \equiv\left\langle\psi_{k}, \xi\right\rangle$.

The normalization is such that $\left\langle\psi_{k}, \phi_{k}\right\rangle=1$. Since (2.11) holds, the solution representation (2.12) is valid and will satisfy $(2.5(b))$, provided that

(d) $c_{k}=0$ if $\operatorname{Re} \lambda_{k}>0$.

Thus we have two other equivalent formulations of the admissible space (2.8) as

(a) $\mathscr{A}(\tau)=\left\{\eta: \eta \in \mathscr{B},\left\langle\psi_{k}, \eta\right\rangle=0\right.$ for all $k$ with $\left.\operatorname{Re} \lambda_{k}>0\right\}$,

(b) $\quad=\operatorname{span}\left\{\phi_{k}: \operatorname{Re} \lambda_{k}<0\right\}$.

These are characterizations of $\mathscr{A}(\tau)$ in terms of the spectrum of $A$. Note that they are independent of $\tau$. When variations with $x$ are allowed in the tail, this may not be the case. We observe that (2.11) and the representation (2.12) make it clear that $S\left(x, x^{\prime}\right)$ has an exponential dichotomy. Our general theory in [11], [12] is formulated in terms of dichotomies and thus the present case serves as an example. In fact, (2.12) leads us to a specific representation of the solution operator'as

$$
S(x, \tau) \xi(\mathbf{y})=\sum_{k} e^{\lambda_{k}(x-\tau)} \phi_{k}(\mathbf{y})\left\langle\psi_{k}, \boldsymbol{\xi}\right\rangle .
$$

** See Gohberg and Krein [8] for a discussion of nonorthogonal bases. 
The completeness (2.11(b)) and the characterization (2.13) allow us to introduce a projection $\mathscr{Q}(\tau)$ which takes $\mathscr{B}$ into $\mathscr{A}(\tau)$ as follows:

(a) $\mathscr{2}(\tau) \mathscr{B}=\mathscr{A}(\tau)$,

where

(b) $\xi \equiv \sum_{k} c_{k}(\xi) \phi_{k} \in \mathscr{B}$

implies

$$
\text { (c) } \mathscr{Q}(\tau) \xi=\sum_{k: \operatorname{Re} \lambda_{k}<0} c_{k}(\xi) \phi_{k} \in \mathscr{A}(\tau) \text {. }
$$

Recalling (2.12(b), (c)), we can write the projection formally as

$$
\text { (d) } \mathscr{2}(\tau) \xi(\mathbf{y})=\sum_{k: \operatorname{Re} \lambda_{k}<0} \phi_{k}(\mathbf{y})\left\langle\psi_{k}, \xi\right\rangle .
$$

Note that the complement of $\mathscr{A}(\tau)$, given by $(I-\mathscr{Q}) \mathscr{B}$, represents all initial data which yield unbounded solutions of (2.6) as $x \rightarrow \infty$. This projection onto the complement of $\mathscr{A}(\tau)$ has the formal representation

$$
[I-\mathscr{Q}(\tau)] \xi(\mathbf{y})=\sum_{k: \operatorname{Re} \lambda_{k}>0} \phi_{k}(\mathbf{y})\left\langle\psi_{k}, \xi\right\rangle .
$$

We can use these projections and the solution operator $S\left(x, x^{\prime}\right)$ to write a general variation of parameters formula for solving the special inhomogeneous form of (2.6):

$$
\begin{aligned}
& \text { (a) } \frac{d \mathbf{w}}{d x}-A \mathbf{w}=\mathbf{F}(x), \quad x>\tau, \mathbf{w} \in \mathscr{B} ; \\
& \text { (b) } \mathscr{2}(\tau) \mathbf{w}(\tau)=\eta \in \mathscr{A}(\tau) \\
& \text { (c) } \lim _{x \rightarrow \infty} \mathbf{w}(x)=\mathbf{0} .
\end{aligned}
$$

The solution representation is

$$
\begin{array}{r}
\mathbf{w}(x)=S(x, \tau) \eta+\int_{\tau}^{x} S\left(x, x^{\prime}\right) \mathscr{Q}\left(x^{\prime}\right) \mathbf{F}\left(x^{\prime}\right) d x^{\prime} \\
-\int_{x}^{\infty} S\left(x, x^{\prime}\right)\left[I-\mathscr{Q}\left(x^{\prime}\right)\right] \mathbf{F}\left(x^{\prime}\right) d x^{\prime} .
\end{array}
$$

This solution is valid provided $\|\mathbf{F}(x)\|<\infty$, since $S\left(x, x^{\prime}\right)$ has an exponential dichotomy. We note that the no-mixing condition holds, that is,

$$
\mathscr{2}(x) S(x, \tau) \xi=S(x, \tau) \mathscr{2}(\tau) \xi
$$

for all $\xi \in \mathscr{B}$ for which $S(x, \tau) \xi$ exists. Note that $(2.17(\mathrm{~b}))$ does not specify $\mathbf{w}(\tau)$ uniquely but only the projection of it into $\mathscr{A}(\tau)$. Using (2.18) and (2.19), we obtain for the complementary part of $\mathbf{w}(\tau)$

$$
[I-\mathscr{Q}(\tau)] \mathbf{w}(\tau)=-\int_{\tau}^{\infty} S\left(\tau, x^{\prime}\right)\left[I-\mathscr{2}\left(x^{\prime}\right)\right] \mathbf{F}\left(x^{\prime}\right) d x^{\prime} .
$$

These are the Asymptotic Boundary Conditions for linear problems. We claim that any solution of $(2.17(\mathrm{a}),(\mathrm{c}))$ must satisfy (2.20). Clearly, any such solution satisfies (2.17(b)) for some $\eta \in \mathscr{A}(\tau)$. Then (2.18) is valid and hence (2.20) follows. Note that this condition is independent of $\eta$ for linear problems. 
Now we return to the nonlinear problem (2.5). If this problem has a solution, we denote it by $\mathbf{w}(x ; \eta)$ where $\eta \in \mathscr{A}(\tau) \subset \mathscr{B}$ is the value taken on at $x=\tau$ of $\mathscr{2} \mathbf{w}(x)$, that is,

$$
\mathscr{2}(\tau) \mathbf{w}(\tau ; \eta)=\eta \in \mathscr{A}(\tau) \text {. }
$$

Then, calling $R(\mathbf{w}(x ; \eta)) \equiv \mathbf{F}(x)$, we see that our solution also satisfies (2.17(a),(c)) and hence (2.20). We rewrite this latter condition for $\mathbf{w}(x ; \eta)$ as

$$
[I-\mathscr{Q}(\tau)] \mathbf{w}(\tau, \eta)=-\int_{\tau}^{\infty} S\left(\tau, x^{\prime}\right)\left[I-\mathscr{Q}\left(x^{\prime}\right)\right] R\left(\mathbf{w}\left(x^{\prime} ; \eta\right)\right) d x^{\prime}
$$

These are the Asymptotic Boundary Conditions for the nonlinear problem. Note that they are highly nonlinear in $\mathbf{w}(\tau)$, the value of the solution at $x=\tau$. In general, (2.21) does not determine $w(\tau)$ uniquely. In fact, it is only a constraint on that part of $\mathbf{w}(\tau)$ that is not in $\mathscr{A}(\tau)$. The freedom left is just enough to enable us to solve a finite problem, on $[0, \tau] \times \Omega$, and to satisfy the conditions (1.1(c)) on the base of the cylinder.*** Under "appropriate" conditions on the nonlinearity it is shown in [11] that unique solutions of (2.17) exist for arbitrary $\eta$ if $\|\eta\|$ is sufficiently small. The proof of existence uses a contraction mapping argument which easily follows from:

Conditions (2.22). (a) There exists a $\delta>0$ and $K<1$ such that for all $\mathbf{v}_{1}(x), \mathbf{v}_{2}(x)$ in $\mathscr{B}[\tau, \infty]$ with $\left\|\mathbf{v}_{1}\right\|<\delta,\left\|\mathbf{v}_{2}\right\|<\delta$ :

$$
\begin{aligned}
\sup _{x>\tau} & \| \int_{\tau}^{x} S\left(x, x^{\prime}\right) \mathscr{Q}\left(x^{\prime}\right)\left[R\left(\mathbf{v}_{1}\left(x^{\prime}\right)\right)-R\left(\mathbf{v}_{2}\left(x^{\prime}\right)\right)\right] d x^{\prime} \\
& -\int_{x}^{\infty} S\left(x, x^{\prime}\right)\left[I-\mathscr{Q}\left(x^{\prime}\right)\right]\left[R\left(\mathbf{v}_{1}\left(x^{\prime}\right)\right)-R\left(\mathbf{v}_{2}\left(x^{\prime}\right)\right)\right] d x^{\prime}\|<K\| \mathbf{v}_{1}-\mathbf{v}_{2} \| .
\end{aligned}
$$

(b) There exists a $\delta_{1} \in(0, \delta)$ such that for all $\mathbf{v}_{1}(x)$ as above:

$$
\begin{aligned}
& \sup _{x>\tau} \| \int_{\tau}^{x} S\left(x, x^{\prime}\right) \mathscr{Q}\left(x^{\prime}\right) R\left(\mathbf{v}_{1}\left(x^{\prime}\right)\right) d x^{\prime} \\
&-\int_{x}^{\infty} S\left(x, x^{\prime}\right)\left[I-\mathscr{Q}\left(x^{\prime}\right)\right] R\left(\mathbf{v}_{1}\left(x^{\prime}\right)\right) d x^{\prime} \|<\delta_{1} .
\end{aligned}
$$

(These are the Assumptions 6.6 of [11] with (c) eliminated, since it follows here for $\|\eta\|$ sufficiently small.)

Specifically, the contraction proceeds as follows:

$$
\text { (a) } \quad \begin{aligned}
& \mathbf{w}^{(1)}(x ; \eta)=S(x ; \tau) \eta, \quad \eta \in \mathscr{A}(\tau) ; \\
& \text { (b) } \quad \mathbf{w}^{(\nu+1)}(x ; \eta)= \\
&
\end{aligned}
$$

Using Conditions 2.22, it is not difficult to show the convergence of these iterates to a solution for $\|\eta\|$ small. We note from (2.23(a),(b)) and (2.19) that

$$
\text { (a) } \mathscr{2}(\tau) \mathbf{w}^{(\nu)}(x ; \eta)=\eta \text { for all } \nu=1,2, \ldots
$$

Thus we must also take as part of the $\mathrm{ABC}$ :

$$
\text { (b) } \mathscr{Q}(\tau) w(\tau, \eta)=\eta \in \mathscr{A}(\tau) \text {. }
$$

*** We do not go into these details here. But existence proofs along the indicated lines can be given and they assume that the "dimensionality" of the base condition is the same as that of $\mathscr{A}(\tau)$. 
Now the finite (in $x$ ) problem that we seek to solve numerically can be summarized, using (2.1) and (2.3), as
(a) $\frac{d \mathbf{w}}{d x}-A \mathbf{w}=\mathbf{R}(\mathbf{w}), \quad 0<x<\tau ;$
(b) $\left[a_{\partial \Omega}(\mathbf{y}) \frac{\partial}{\partial \nu}+b_{\partial \Omega}(\mathbf{y})\right] w_{1}(x, \mathbf{y})=0$,
$0<x<\tau, \mathbf{y} \in \partial \Omega($ or $\mathbf{w}(x) \in \mathscr{B}) ;$
(c) $b_{1}(\mathbf{y}) w_{2}(0)+b_{0}(\mathbf{y}) w_{1}(0)=c_{0}(\mathbf{y})-b_{0}(\mathbf{y}) u_{\infty}(\mathbf{y}), \quad y \in \Omega$;
(d) $\mathscr{Q}(\tau) \mathbf{w}(\tau)=\eta$;
(e) $[I-\mathscr{Q}(\tau)] \mathbf{w}(\tau, \boldsymbol{\eta})=-\int_{\tau}^{\infty} S\left(\tau, x^{\prime}\right)\left[I-\mathscr{Q}\left(x^{\prime}\right)\right] \mathbf{R}\left(\mathbf{w}\left(x^{\prime}, \eta\right)\right) d x^{\prime}$.

2.1. Approximations to the $A B C$. Of course, in most problems we cannot expect to know exactly the projection, $2(\tau)$, or the solution operator, $S\left(x, x^{\prime}\right)$. Further, the exact solution $\mathbf{w}(x, \eta)$ in the tail, $x>\tau$, is also not known. Thus we must approximate these operators and the solution in the tail in order to apply the ABC in $(2.25(\mathrm{~d}),(\mathrm{e}))$.

When $\mathscr{Q}(\tau)$ and $S\left(x, x^{\prime}\right)$ are approximated, it is clear how to modify the ABC - we simply use these approximations in $(2.25(\mathrm{~d}),(\mathrm{e}))$. This is illustrated in Section 3.

To get around the unknown solution in the tail, $\mathbf{w}(x, \eta)$, we can use the iteration scheme $(2.23(\mathrm{a}),(\mathrm{b}))$ and terminate it at some finite order, $m$. Then, in place of $(2.25(\mathrm{e}))$, we use

$$
[I-\mathscr{Q}(\tau)] \mathbf{w}(\tau, \eta)=-\int_{\tau}^{\infty} S(\tau, x)[I-\mathscr{Q}(x)] \mathbf{R}\left(\mathbf{w}^{(m)}(x, \eta)\right) d x .
$$

We call these the ABC of order $m$. Obviously, to use them, the "correct" value of $\eta \in \mathscr{A}(\tau)$ is assumed known. In constructive procedures, as we show later in our numerical work, the value of $\eta$ is determined iteratively along with the rest of $\mathbf{w}(x)$ over $0 \leqslant x \leqslant \tau$. Then $\mathbf{w}^{(m)}(x, \eta)$ for $x>\tau$, in the tail, must also be determined for each iterate, to use in (2.26).

However, there is an alternative way to proceed, based on the assumption that $\|\mathbf{w}(x)\|$ in the tail is small. We simply expand $\mathbf{R}(\mathbf{w})$ in a Taylor series about $\mathbf{w}=\mathbf{0}$ and retain a finite number of terms. It is not difficult to see that, if we retain only the terms up to and including order $m$, the error in this procedure is of the same order as that in using (2.26). We express this new form of the $\mathrm{ABC}$ of order $m$ in terms of the expansion coefficients for a spectral representation of the solution in terms of eigenfunctions introduced in (2.9). (When this eigenvalue problem is not selfadjoint we must employ the adjoint eigenproblem to carry out the expansions.) We indicate the details of this alternative procedure below.

The Taylor expansion of $\mathbf{R}(\mathbf{w})$ is, in terms of the higher-order Fréchet derivatives $\mathbf{R}_{w w}$, etc.,

$$
\text { (a) } \mathbf{R}(\mathbf{w})=\frac{1}{2} \mathbf{R}_{w w}(0) \mathbf{w w}+\frac{1}{6} \mathbf{R}_{w w w}(0) \mathbf{w w w}+\cdots
$$

Using the expansion

$$
\text { (b) }\left\{\begin{array}{l}
\mathbf{w}(x, \boldsymbol{\eta})=\sum_{j} C_{j}(x, \boldsymbol{\eta}) \phi_{j}, \\
C_{j}(x, \boldsymbol{\eta})=\left\langle\psi_{j}, \mathbf{w}\right\rangle,
\end{array}\right.
$$


we have

$$
\text { (c) } \mathbf{R}(\mathbf{w})=\frac{1}{2} \sum_{i, j} \mathbf{R}_{w w}(0) C_{i} C_{j} \phi_{i} \phi_{j}+\frac{1}{6} \sum_{i, j, k} \mathbf{R}_{w w w}(0) C_{i} C_{j} C_{k} \phi_{i} \phi_{j} \phi_{k}+\cdots
$$

However, an eigenfunction expansion of $\mathbf{R}(\mathbf{w})$ in the form

$$
\text { (a) } \mathbf{R}(\mathbf{w})=\sum_{n} \rho_{n}(\mathbf{w}) \phi_{n}
$$

with

$$
\rho_{n}(\mathbf{w})=\left\langle\psi_{n}, \mathbf{R}(\mathbf{w})\right\rangle
$$

yields, on using (2.27c),

$$
\text { (b) } \rho_{n}(\mathbf{w})=\sum_{i, j} \alpha_{i j}^{(n)} C_{i} C_{j}+\sum_{i, j, k} \alpha_{i j k}^{(n)} C_{i} C_{j} C_{k}+\cdots
$$

Here we have introduced

$$
\begin{aligned}
& \alpha_{i j}^{(n)} \equiv\left\langle\psi_{n}, \frac{1}{2} \mathbf{R}_{w w}(0) \phi_{i} \phi_{j}\right\rangle, \\
& \alpha_{i j k}^{(n)} \equiv\left\langle\psi_{n}, \frac{1}{6} \mathbf{R}_{w w w}(0) \phi_{i} \phi_{j} \phi_{k}\right\rangle,
\end{aligned}
$$

Note that the explicit dependence of $\mathbf{R}(\mathbf{w}(x, \eta))$ on $\mathbf{w}$ in the expansion (2.28) is all contained in the coefficients $C_{j}(x, \eta)$ of $(2.27(\mathrm{~b}))$. The coefficients $\alpha_{i j}^{(n)}, \alpha_{i j k}^{(n)}, \ldots$ can, in principle, be determined once and for all. Then they are used with any iterates approximating $\mathbf{w}$ by simply recomputing the new $C_{j}(x, \eta)$ to get new approximations to $\mathbf{R}(\mathbf{w}(x, \eta))$.

We use the expansions (2.27(b)) and (2.28) in the $\mathrm{ABC}(2.25(\mathrm{e}))$ to get, with the aid of (2.14) and (2.16),

$$
\begin{aligned}
& C_{n}(\tau, \boldsymbol{\eta})=-\int_{\tau}^{\infty} e^{\lambda_{n}(\tau-x)}\left\{\sum_{i, j} \alpha_{i j}^{(n)} C_{i}(x, \boldsymbol{\eta}) C_{j}(x, \boldsymbol{\eta})\right. \\
&\left.+\sum_{i, j, k} \alpha_{i j k}^{(n)} C_{i}(x, \boldsymbol{\eta}) C_{j}(x, \boldsymbol{\eta}) C_{k}(x, \boldsymbol{\eta})+\cdots\right\} d x
\end{aligned}
$$

for all $n$ with $\operatorname{Re} \lambda_{n}>0$.

This form of the $\mathrm{ABC}$ is no more useful than using (2.27(a)) in (2.25(e)). However, if we wish to truncate this form of the $\mathrm{ABC}$ after quadratic terms, we can use for the $C_{j}(x, \eta)$ on the right-hand side the coefficients obtained by expanding $\mathbf{w}^{(1)}(x ; \eta)$ from (2..23(a)). From (2.14) and (2.15(d)) these coefficients are found to be

$$
\text { (a) } \begin{aligned}
C_{j}(x, \eta) & =e^{\lambda_{j}(x-\tau)} C_{j}(\tau, \eta) & & \text { if } \operatorname{Re} \lambda_{j}<0 \\
& =0 & & \text { if } \operatorname{Re} \lambda_{j}>0
\end{aligned}
$$

where

$$
\text { (b) } C_{j}(\tau, \eta)=\left\langle\psi_{j}, \eta\right\rangle \equiv c_{j}
$$

or equivalently,

$$
\eta=\sum_{k: \operatorname{Re} \lambda_{k}<0} c_{k} \phi_{k} \in \mathscr{A}(\tau) .
$$


With (2.30) in (2.29) we thus get, on retaining only quadratic terms and using $C_{n}(\tau, \eta) \equiv c_{n}$,

$$
c_{n}=\sum_{\substack{i, j: \operatorname{Re} \lambda_{i}<0 \\ \operatorname{Re} \lambda_{j}<0}} \frac{\alpha_{i j}^{(n)} c_{i} c_{j}}{\lambda_{\Lambda}+\lambda_{j}-\lambda_{n}}, \text { for all } n \text { with } \operatorname{Re} \lambda_{n}>0 .
$$

The above second-order form of the $\mathrm{ABC}$ are quite useful and explicit; that is, no quadratures in $x$ are involved. Of course, in order to use them, we must be prepared to find the eigenfunction representation, that is, to compute the $c_{j}$ as in $(2.30(\mathrm{~b}))$, and then given the $c_{n}$ as in (2.31) we must form $\mathbf{w}(\tau, \eta)$ as in $(2.27(\mathrm{~b}))$. Thus the use of fast transforms can play an important role in the efficient implementation of our procedures.

2.2. The Quadratic $A B C$ in the Selfadjoint Case. When $L_{1} \equiv 0$, the eigenvalue problems (2.9) and (2.10) are selfadjoint. This case occurs very often in applications and so we discuss it here.

A glance at (2.10) reveals that now $\lambda_{k}^{2}, k=1,2, \ldots$, are the eigenvalues of a second-order selfadjoint elliptic operator. In order to satisfy (2.11(a)), all of these $\lambda_{k}^{2}$ must be positive. Then each eigenfunction $\phi_{k}(\mathbf{y})$ of (2.10) belongs to a pair of eigenvalues, $\pm \lambda_{k}$, of (2.9). The eigenfunctions of (2.9) are as in (2.10(c)), and to label them all we simply use $k= \pm 1, \pm 2, \ldots$ and

$$
\text { (a) } \quad \lambda_{ \pm k}= \pm \sqrt{\lambda_{k}^{2}}, \quad \lambda_{k}=-\lambda_{-k}>0, \quad \phi_{ \pm k}(\mathbf{y})=\left(\begin{array}{c}
1 \\
\lambda_{ \pm k}
\end{array}\right) \phi_{k}(\mathbf{y})
$$

For the $\psi_{k}(\mathbf{y})$ we take

$$
\text { (b) } \psi_{ \pm k}(\mathbf{y})= \pm\left(\begin{array}{c}
\lambda_{ \pm k} \\
1
\end{array}\right) \phi_{k}(\mathbf{y}), \quad k=1,2, \ldots ;
$$

and the $\phi_{k}(\mathbf{y})$ are normalized by

$$
\text { (c) }\left\|\phi_{k}\right\|^{2}=\int \ldots \int \phi_{k}^{2}(\mathbf{y}) d \mathbf{y}=\frac{1}{2 \lambda_{k}}, \quad k=1,2, \ldots
$$

Then $\left\langle\psi_{j}, \phi_{k}\right\rangle=\delta_{j k}$ still holds, which accounts for the sign choice in (2.32(b)) and the normalization in $(2.32(\mathrm{c}))$. We have the eigenfunction expansions as in (2.27(b)). However, with $\mathbf{w} \equiv\left(\begin{array}{l}v \\ v_{x}\end{array}\right)$, we note that

$$
\left.\begin{array}{l}
\text { (a) } c_{k}=\left\langle\psi_{k}, \mathbf{w}\right\rangle=\lambda_{k}\left(v, \phi_{k}\right)+\left(v_{x}, \phi_{k}\right) \\
\text { (b) } c_{-k}=\left\langle\psi_{-k}, \mathbf{w}\right\rangle=\lambda_{k}\left(v, \phi_{k}\right)-\left(v_{x}, \phi_{k}\right)
\end{array}\right\}, \quad k=1,2, \ldots
$$

Here we introduce for $v(x, y) \in W_{2}^{0}(\Omega)$ on $x>0$ :

$$
\text { (c) } d_{k}(x) \equiv\left(v, \phi_{k}\right) \equiv \int{ }_{\Omega} \int v(x, \mathbf{y}) \phi_{k}(\mathbf{y}) d y \text {, }
$$

and note that under appropriate smoothness:

$$
\text { (d) } \frac{d}{d x} d_{k}(x) \equiv d_{k}^{\prime}(x)=\left(v_{x}, \phi_{k}\right) \text {. }
$$

Of course, eigenfunction expansions of $v(x, \mathbf{y})$ and $v_{x}(x, \mathbf{y})$ are given by

$$
\begin{aligned}
& \text { (a) } v(x, \mathbf{y}) \approx \sum_{k=1}^{\infty} 2 \lambda_{k} d_{k}(x) \phi_{k}(\mathbf{y}), \\
& \text { (b) } v_{x}(x, \mathbf{y}) \approx \sum_{k=1}^{\infty} 2 \lambda_{k} d_{k}^{\prime}(x) \phi_{k}(\mathbf{y}) .
\end{aligned}
$$


To employ the above results in the second-order $\mathrm{ABC}$ of $(2.31)$, we recall that by our labeling in (2.32(a)):

$$
\operatorname{Re} \lambda_{k}>0, \quad \operatorname{Re} \lambda_{-k}<0 \text { for } k=1,2, \ldots
$$

Thus we get, using (2.33) in (2.31),

$$
\begin{aligned}
d_{n}^{\prime}(\tau)= & -\lambda_{n} d_{n}(\tau) \\
& -\sum_{\substack{i>0 \\
j>0}} \frac{\alpha_{-i,-j}^{(n)}}{\lambda_{i}+\lambda_{j}+\lambda_{n}}\left[\lambda_{i} d_{i}(\tau)-d_{i}^{\prime}(\tau)\right]\left[\lambda_{j} d_{j}(\tau)-d_{j}^{\prime}(\tau)\right], \\
& n=1,2, \ldots
\end{aligned}
$$

For practical applications we renormalize the $\alpha$-coefficients and write them as ${ }^{\dagger}$

$$
\text { (a) } \quad \beta_{i j}^{(n)} \equiv \frac{4 \lambda_{i} \lambda_{j} \alpha_{i, j}^{(n)}}{\lambda_{i}+\lambda_{j}+\lambda_{n}} ; \quad n, i, j=1,2, \ldots
$$

Then we get from (2.35), retaining second-order accuracy, the conditions

$$
\text { (b) } d_{n}^{\prime}(\tau)=-\lambda_{n} d_{n}(\tau)-\sum_{\substack{i>0 \\ j>0}} \beta_{i j}^{(n)} d_{i}(\tau) d_{j}(\tau)
$$

These are the quadratic $\mathrm{ABC}$ that we employ in the calculations reported in Section 4. We derive $(2.36(\mathrm{~b}))$ by using the leading-order term in $(2.35)$, that is, we set $d_{k}^{\prime}(\tau)=-\lambda_{k} d_{k}(\tau)$ in the quadratic term, and hence second-order accuracy is retained.

3. Discrete Approximations. We now show how to employ the Asymptotic Boundary Conditions (of second or lower order) in a numerical computation. For simplicity we assume the selfadjoint case, $L_{1} \equiv 0$, no mixed derivatives in $L_{2}$ (i.e., $a_{i j}=0$ for $i \neq j$ ), domain bounded by coordinate surfaces and Dirichlet boundary conditions (i.e., $a_{\partial \Omega} \equiv 0$ ). We also assume a uniform grid $\Omega_{h}$ with mesh $h$ placed on $\Omega$. Then $L_{2}$ is approximated by the usual centered second-order $(2 N+1)$-point scheme, say,

$$
L_{2}^{h} \phi^{h}(\mathbf{y}) \equiv \sum_{j=1}^{N} D_{j}^{+}\left[a_{j j}\left(\mathbf{y}-\frac{h}{2} \mathbf{e}_{j}\right) D_{j}^{-} \phi^{h}(\mathbf{y})\right]+a(\mathbf{y}) \phi^{h}(\mathbf{y}) .
$$

Here, $D_{j}^{ \pm}$are the forward and backward finite-difference operators in the $y_{j}$ th coordinate direction, $\mathbf{e}_{j}$ is the unit vector in the $y_{j}$ th coordinate direction, $\mathbf{y}$ is any point in the interior of $\Omega_{h}$, call it $\Omega_{h}^{0}$, and $\phi^{h}(\mathbf{y})$ is any mesh function defined on $\Omega_{h}$. On the $x$-interval $[-h / 2, \tau+h / 2]$ we also use the spacing $h$ to place a grid of points $x_{j}=j h-h / 2,0 \leqslant j \leqslant J+1$, so that $\tau=J h$. Then, if $v_{h}(x, \mathbf{y})$ is a mesh function on $\left\{x_{j}\right\} \times \Omega_{h}$ which is to approximate $v(x, \mathbf{y})$, we use as the difference equations to approximate $(2.25(\mathrm{a}))$ :

$$
D_{x}^{+} D_{x}^{-} v^{h}\left(x_{j}, \mathbf{y}\right)+L_{2}^{h} v^{h}\left(x_{j}, \mathbf{y}\right)=r\left(v^{h}\left(x_{j}, \mathbf{y}\right)\right), \quad 1 \leqslant j \leqslant J .
$$

In defining $v$ and $r(\cdot)$ above we have to use an approximation to $u_{\infty}(\mathbf{y})$, the solution of the limiting problem (1.3). We use for this the solution of the discrete problem

$$
\begin{aligned}
& \text { (a) } L_{2}^{h} u_{\infty}^{h}(\mathbf{y})=f\left(u_{\infty}^{h}(\mathbf{y}), \mathbf{y}\right), \quad \mathbf{y} \in \Omega_{h}^{0} ; \\
& \text { (b) } u_{\infty}^{h}(\mathbf{y})=c_{\partial \Omega}(\mathbf{y}), \quad \mathbf{y} \in \partial \Omega_{h} .
\end{aligned}
$$

\footnotetext{
${ }^{\dagger}$ Here we have used the fact that in the selfadjoint case $\phi_{-j}(\mathbf{y}) \equiv \phi_{j}(\mathbf{y})$, and so $\alpha_{-i,-j}^{(n)}=\alpha_{i, j}^{(n)}$.
} 
Here we have indicated the Dirichlet boundary conditions to be imposed on $\partial \Omega$. This nonlinear difference problem is to be solved by some iterative procedure, preferably Newton's method or some close variant. When $u_{\infty}^{h}(\mathbf{y})$ is determined, then $r(\cdot)$ is defined as in (2.2(b)) and of course (2.1) also is applied on $\Omega_{h}$, so that

$$
\text { (c) } v^{h}\left(x_{j}, \mathbf{y}\right) \equiv u^{h}\left(x_{j}, \mathbf{y}\right)-u_{\infty}^{h}(\mathbf{y}), \quad \mathbf{y} \in \Omega_{h} .
$$

The boundary conditions (1.1(c)) or, equivalently, (2.25(c)), are imposed at $x=0$ and use values at $x_{0}=-h / 2$ and $x_{1}=h / 2$ :

$$
\begin{gathered}
b_{1}(\mathbf{y})\left[\frac{v^{h}\left(x_{1}, \mathbf{y}\right)-v^{h}\left(x_{0}, \mathbf{y}\right)}{h}\right]+b_{0}(\mathbf{y})\left[\frac{v^{h}\left(x_{1}, \mathbf{y}\right)+v^{h}\left(x_{0}, \mathbf{y}\right)}{2}\right] \\
=c_{0}(\mathbf{y})-b_{0}(\mathbf{y}) u_{\infty}^{h}(\mathbf{y}), \quad \mathbf{y} \in \Omega_{h}^{0} .
\end{gathered}
$$

Of course, if $b_{1}(\mathbf{y}) \equiv 0$, we need not use this centering procedure. Then we use $[0, \tau+h / 2]$ as the $x$-interval with $x_{0}=0, x_{j}=j h$, and $\tau=\left(J+\frac{1}{2}\right) h$.

On the lateral boundary we use the Dirichlet conditions which, by (1.1(b)) with $a_{\partial \Omega} \equiv 0$ and (3.3(b), (c)), become

$$
v^{h}\left(x_{j}, \mathbf{y}\right)=0, \quad \mathbf{y} \in \partial \Omega_{h}, 1 \leqslant j \leqslant J .
$$

Finally, we turn to the discrete version of the ABC's. In order to employ them we must approximate the projection $2(\tau)$ or, equivalently, we must approximate the solutions of the eigenvalue problem. In the present selfadjoint case, the discrete eigenproblem and normalization corresponding to (2.32(c)) are

$$
\begin{aligned}
& \text { (a) } L_{2}^{h} \phi_{k}^{h}(\mathbf{y})+\left(\lambda_{k}^{2}-f_{u}\left(u_{\infty}^{h}(\mathbf{y})\right) \phi_{k}^{h}(\mathbf{y})=0, \quad \mathbf{y} \in \Omega_{h}^{0} ;\right. \\
& \text { (b) } \phi_{k}^{h}(\mathbf{y})=0, \quad \mathbf{y} \in \partial \Omega_{h} ; \\
& \text { (c) } \sum_{\mathbf{y} \in \Omega_{h}^{0}}\left[\phi_{k}^{h}(\mathbf{y})\right]^{2} h^{N}=\frac{1}{2 \lambda_{k}}
\end{aligned}
$$

We denote the number of meshpoints in $\Omega_{h}^{0}$ by $\hat{K}(h)$ and then $1 \leqslant k \leqslant \hat{K}(h)$ in (3.6). It is well known that, for a sufficiently fine mesh, some of the eigenvalues and eigenvectors of (3.6) are $\mathcal{O}\left(h^{2}\right)$ approximations to the corresponding eigenpairs of (2.10). Say, those for $k=1,2, \ldots, K(h)<\hat{K}(h)$, where $K(h) \rightarrow \infty$ as $h \rightarrow 0$. The spectral resolution of the mesh function $v^{h}\left(x_{j}, \mathbf{y}\right)$ can be written as

$$
\text { (a) } v^{h}\left(x_{j}, \mathbf{y}\right)=\sum_{k=1}^{K(h)} 2 \lambda_{k}^{h} d_{k}\left(x_{j}\right) \phi_{k}^{h}(\mathbf{y})
$$

where

$$
\text { (b) } d_{k}^{h}\left(x_{j}\right)=\sum_{\mathbf{y} \in \Omega_{h}^{0}} v^{h}\left(x_{j}, \mathbf{y}\right) \phi_{k}^{h}(\mathbf{y}) h^{N} .
$$

Of course, the $\beta_{i j}^{(n)}$ of $(2.36(\mathrm{a}))$ are approximated in the obvious way, using in analogy with $(2.28(\mathrm{c}))$,

$$
\boldsymbol{\alpha}_{i, j}^{(n) h} \equiv \sum_{\mathbf{y} \in \Omega_{h}^{0}} \frac{1}{2} f_{u u}\left(u_{\infty}^{h}(\mathbf{y})\right) \phi_{n}^{h} \phi_{i}^{h}(\mathbf{y}) \phi_{j}^{h}(\mathbf{y}) h^{N} .
$$


Now a discrete form of the second-order ABC, (2.36(b)), can be written at $\tau$, the midpoint of the last $x$-interval $\left[x_{J}, x_{J+1}\right]$, as

$$
\begin{aligned}
& \frac{d_{n}^{h}\left(x_{J+1}\right)-d_{n}^{h}\left(x_{J}\right)}{h} \\
& =-\lambda_{n} \frac{d_{n}^{h}\left(x_{J+1}\right)+d_{n}^{h}\left(x_{J}\right)}{2} \\
& \quad-\sum_{i=1}^{K(h)} \sum_{j=1}^{K(h)} \beta_{i j}^{(n) h}\left[\frac{d_{i}^{h}\left(x_{J+1}\right) d_{j}^{h}\left(x_{J+1}\right)+d_{i}^{h}\left(x_{J}\right) d_{j}^{h}\left(x_{J}\right)}{2}\right], \\
& n=1,2, \ldots, \hat{K}(h) .
\end{aligned}
$$

Combined with (3.7), these form nonlinear boundary conditions on the mesh function $v^{h}\left(x_{j}, \mathbf{y}\right)$. The complete finite, nonlinear, approximating problem consists of (3.2), (3.4), (3.5) and (3.9). Fortunately, we need not take $K(h)=\hat{K}(h)$, the number of points in $\Omega_{h}^{0}$, as not all of these eigenfunctions are reasonable approximations to their continuous counterparts. The choice of $K(h)$ is not too crucial in most applications.

We point out another way to formulate the second-order ABC. Using (2.34) we get, on multiplying (2.36(b)) by $2 \lambda_{n} \phi_{n}(\mathbf{y})$ and summing on $n$,

$$
\text { (a) } v_{x}(\tau, \mathbf{y})=-\sum_{n>0} 2 \lambda_{n}^{2} d_{n}(\tau) \phi_{n}(\mathbf{y})-\sum_{\substack{i>0 \\ j>0 \\ n>0}} 2 \lambda_{n} \beta_{i j}^{(n)} d_{i}(\tau) d_{j}(\tau) \phi_{n}(\mathbf{y})
$$

Or, in terms of $v$ and $v_{x}$, this is

$$
\begin{aligned}
v_{x}(\tau, \mathbf{y})= & -\sum_{n>0} 2 \lambda_{n}^{2}\left(v(\tau, \cdot), \phi_{n}\right) \phi_{n}(\mathbf{y}) \\
& -\sum_{\substack{i>0 \\
j>0 \\
n>0}} 2 \lambda_{n} \beta_{i j}^{(n)}\left(v(\tau, \cdot), \phi_{i}\right)\left(v(\tau, \cdot), \phi_{j}\right) \phi_{n}(\mathbf{y}) .
\end{aligned}
$$

The nonlocal nature of our boundary conditions with respect to the artificial boundary, $\Omega$, at $x=\tau$ is clearly indicated by the inner products $\left(v, \phi_{k}\right)$ in (3.10). Obviously, in the numerical applications we replace (3.10) by centering discrete quantities at $x=\tau$ using values at $x_{J}$ and $x_{J+1}$, essentially as in (3.9), but using (3.7(b)) in (3.10(a)) to approximate the $d_{j}(\tau)$ by $d_{j}^{h}(\tau)$. These forms of the ABC make it clear that we do not have to use all $\hat{K}(h)$ eigenfunctions of the discrete eigenproblem (3.6) in order to have a determined system (i.e., as many unknowns as equations).

Whichever discrete form of the ABC's is used, we must solve the final discrete problem by some iterative procedure (in the nonlinear case). To use Newton's method, we must solve linear systems with coefficient matrix given by the Jacobian matrix of the full nonlinear problem. From (3.2), the derivatives with respect to the unknown, $v^{h}\left(x_{j}, \mathbf{y}\right)$ for all $\mathbf{y} \in \Omega_{h}$, form a banded (in fact, block bidiagonal) matrix with bandwidth essentially equal to the number of points in $\Omega_{h}-$ say $M^{N}$ if $\Omega$ is a cube in $\mathbf{R}^{N}$ with $M$ points on each edge. The boundary conditions (3.4) and (3.5) do not alter this structure, but the $\mathrm{ABC}$ as in (3.9) or the discrete form of (3.10) may. At worst, they couple the last $2 M^{N}$ columns, thus increasing the bandwidth by $M^{N}$. If this is the case, we use a solution algorithm based on "bordering" or block Gaussian elimination and so need not factor a matrix with bandwidth greater than $M^{N}+1$. 
The effect of our nonlocal boundary conditions on iterative methods for solving the linear systems that arise, either from Newton's method in the nonlinear case or the basic linear difference equations from linear problems, has not been examined in any generality. An equivalent of the linear form of $(3.10(b))$ has been used by Bayliss, Goldsteín and Turkel [5], and they find essentially no degradation of a preconditioned conjugate gradient algorithm for the Helmholtz equation in $N=3$ dimensions.

Of course, using the full quadratic ABC's as in (3.9) or (3.10) requires a significant number of additional calculations just to evaluate the matrix elements in the Jacobian. For example, the discrete form of (3.10(b)) would yield matrix elements of the form

$$
\text { (a) } 2(\mathbf{y}, \hat{\mathbf{y}})=\sum_{\mathbf{y}^{\prime} \in \Omega_{h}} A\left(\mathbf{y}, \hat{\mathbf{y}}, \mathbf{y}^{\prime}\right) v^{h}\left(\tau, \mathbf{y}^{\prime}\right),
$$

where

$$
\text { (b) } A\left(\mathbf{y}, \hat{\mathbf{y}}, \mathbf{y}^{\prime}\right)=\sum_{i, j, k}^{K(h)} 2 \lambda_{k} \beta_{i j}^{(k)} \phi_{i}^{h}(\hat{\mathbf{y}}) \phi_{j}^{h}\left(\mathbf{y}^{\prime}\right) \phi_{k}^{h}(\mathbf{y}) h^{N} .
$$

The $A\left(\mathbf{y}, \hat{\mathbf{y}}, \mathbf{y}^{\prime}\right)$ can be evaluated in advance, and so only (3.11(a)) need be done each iteration. Since it must be done for each $\mathbf{y}$ and $\hat{\mathbf{y}} \in \Omega_{h}$, this involves $\mathcal{O}\left(M^{3 N}\right)$ multiplications per iteration. The direct solution of the banded system requires essentially $J M^{3 N}$ multiplications.

There are two ways in which the error due to the $\mathrm{ABC}$ can be reduced: first, by taking more terms in the expansion (i.e., higher-order $A B C$ 's); second, by moving the location of the boundary, $\tau$, further out. The latter requires essentially $\mathcal{O}\left(\Delta J M^{3 N}\right)$ additional multiplications to move $\tau$ out to $\tau+h \Delta J$. Using an approximation of order $s$, rather than just second order, changes the operation count to evaluate the new form of $(3.11(\mathrm{a}))$ to $\mathcal{O}\left(M^{(s+1) N}\right)$. Thus it appears that our choice $s=2$ is in some sense optimal if direct solvers are to be used.

4. Example: Bratu Problem in a Stepped Channel. We consider the Bratu equation

$$
\Delta u \equiv \frac{\partial^{2} u}{\partial x^{2}}+\frac{\partial^{2} u}{\partial y^{2}}=-\kappa e^{u}, \quad(x, y) \in D,
$$

subject to Dirichlet conditions, say,

$$
u=0, \quad(x, y) \in \partial D .
$$

Such problems arise in the theory of thermal ignition of gases (see Aris [3]), and Eq. (4.1) is frequently associated with the names of Gelfond and Frank-Kamenetskii. Existence of positive solutions of (4.1)-(4.2) on finite domains, $D$, has been studied by many authors.

We shall consider the Bratu problem (4.1)-(4.2) on infinite domains consisting of a horizontal channel with a rectangular region cut out of, or added onto the channel, along one of its sides. Thus we take $\bar{D}$, the closure of $D$, to be a stepped channel given by

$$
\begin{aligned}
\bar{D} \equiv & \left\{(x, y):\left|x-x_{0}\right| \geqslant a, 0 \leqslant y \leqslant 1\right\} \\
& \cup\left\{(x, y):\left|x-x_{0}\right| \leqslant a, d \leqslant y \leqslant 1\right\} .
\end{aligned}
$$

For $d \in(0,1)$ the channel is narrowed along $x_{0}-a<x<x_{0}+a$, while for $d<0$ it is wider there; see Figure 1. 


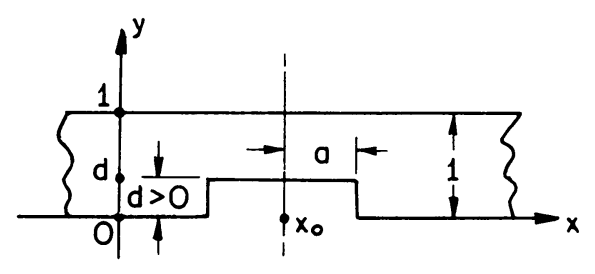

(a)

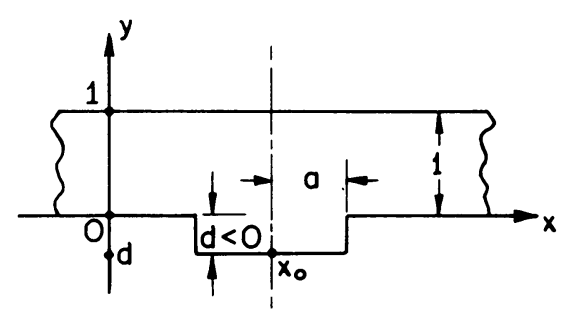

(b)

FIGURE 1

The stepped channel with: (a) $d>0$; (b) $d<0$.

The boundary conditions (4.2) apply at all finite boundary points, while at $\infty$ we impose

$$
\lim _{|x| \rightarrow \infty} u(x, y)=u_{\infty}(y) .
$$

Here, $u_{\infty}(y)$ is a solution of the limiting cross-sectional problem:

$$
\begin{aligned}
& \text { (a) } \frac{d^{2} u_{\infty}(y)}{d y^{2}}=-\kappa e^{u_{\infty}(y)}, \quad 0<y<1 ; \\
& \text { (b) } u_{\infty}(0)=u_{\infty}(1)=0 .
\end{aligned}
$$

This is just the one-dimensional Bratu problem on the unit interval, and it can be solved, implicitly, in closed form to get

$$
\text { (a) } u_{\infty}(y)=\ln \left[\frac{e^{m}}{\cosh ^{2} \alpha\left(y-\frac{1}{2}\right)}\right]
$$

where

$$
\text { (b) } \alpha \equiv \sqrt{e^{m} \kappa / 2}, \quad m=u_{\infty}\left(\frac{1}{2}\right) \text {. }
$$

To satisfy the boundary conditions, $\kappa$ and $m$ must be related by

$$
e^{m}=\cosh ^{2} \alpha / 2 \text {. }
$$

A little manipulation using $\cosh ^{-1} z$ reveals that this can be put into the form

$$
\text { (c) } \sqrt{\frac{\kappa}{2}}=F(\theta) \equiv \sqrt{\theta} \ln \left(\frac{1+\sqrt{1-\theta}}{1-\sqrt{1-\theta}}\right), \quad 0<\theta \equiv e^{-m}<1 .
$$

It is easy to see that $F(0)=F(1)=0$ and $F(\theta)$ has one maximum over $0 \leqslant \theta \leqslant 1$ at $\theta=\theta^{*}$, the root of $F^{\prime}(\theta)=0$, or

$$
\text { (a) } \ln \left(\frac{1+z}{1-z}\right)=\frac{1}{z}, \quad \text { with } z \equiv \sqrt{1-\theta}
$$

Thus, we easily find that $(4.6(\mathrm{c}))$ has two roots, $\theta_{L}$ and $\theta_{U}$ in $0<\theta_{L}<\theta_{*}<\theta_{U}<1$, for each $\kappa$ in $0<\kappa<\kappa^{*}$, where

$$
\text { (b) } \kappa^{*}=2 F^{2}\left(\theta^{*}\right)=3.5138 \ldots
$$

There are no real roots for $\kappa$ outside $\left[0, \kappa^{*}\right]$ and hence (4.5) has no real solutions for $\kappa>\kappa^{*}$. 
We must use the solution with $\theta=\theta_{L}(\kappa)$ in (4.4) so that condition (2.11(a)) is satisfied. That is, if we use for $u_{\infty}(y)$ the minimal positive solution of $(4.5)$, then it follows from Theorem 5.1 in Keller and Cohen [16] that the eigenvalues $\lambda_{k}^{2}$ of (2.10) are all positive. (If we use $\theta=\theta_{U}(\kappa)$, it can be shown that $\lambda_{1}^{2}<0$, and our theory does not apply. We believe that in this case the behavior as $|x| \rightarrow \infty$ can be oscillatory, and this is currently under study.) In our actual calculations we do not employ the forms given in (4.6), but rather we solve (4.5) numerically via finite differences.

Finally, we seek only solutions of (4.1) which are symmetric in $x$ about $x_{0}$. Thus we need only consider $D_{>}$, the semi-infinite part of $D$ in which $x>x_{0}$, say. Along the new boundary at $x=x_{0}$ we impose the symmetry condition

$$
\frac{\partial u}{\partial x}\left(x_{0}, y\right)=0, \quad d<y<1 \text {. }
$$

For the numerical solution of our problem we introduce an artificial boundary at several values of $x=\tau>x_{0}+a$. At each such boundary we have employed three different asymptotic boundary conditions:

(i) Zero-order conditions:

$$
\begin{aligned}
& \partial u(\tau, y) / \partial x=0 ; \\
& d_{n}^{\prime}(\tau)+\lambda_{n} d_{n}(\tau)=0
\end{aligned}
$$

(ii) First-order (linear) approximation:

(iii) Second-order (quadratic) approximation: see Eq. (2.36).

To insure accuracy near the corners in the domain $D_{>}$, we first map it into a straight channel before discretization. To do this, we use the conformal mapping, for $d>0$ :

(a) $\zeta \equiv \xi+i \eta=F^{-1}(z) \equiv F^{-1}(x+i y)$;

(b) $F(\zeta ; d) \equiv \frac{2}{\pi}\left\{\ln \left[w^{1 / 2}+(w+\alpha)^{1 / 2}\right]\right.$

$$
\begin{gathered}
\left.-(1-\alpha)^{1 / 2} \ln \left[(w+\alpha)^{1 / 2}+(1-\alpha)^{1 / 2} w^{1 / 2}\right]\right\} \\
+(1-\alpha)^{1 / 2} \zeta \\
\text { (c) } w \equiv e^{\pi \zeta}-1, \quad \alpha \equiv(2-d) d .
\end{gathered}
$$

For $d<0$, the mapping is obtained by setting $d^{*}=d(d-1)^{-1}$ and replacing $F(\zeta ; d)$ by $F^{*}(\zeta ; d)$, where

$$
F^{*}(\zeta ; d) \equiv\left(d^{*}-1\right)^{-1} \bar{F}\left(-\bar{\zeta} ; d^{*}\right)+d .
$$

Now the straight boundaries at $x=x_{0}$ and $x=\tau$ are mapped into slightly curved boundaries in the $(\xi, \eta)$-plane. This yields perturbations to the boundary conditions, and they are easily calculated using linear interpolation on the net functions which approximate the solution.

In the new domain in the $(\xi, \eta)$-plane, a straight channel with slightly curved ends, the Bratu equation (4.1) is approximated by the usual centered differences on a uniform grid with mesh $h=0.05$. Newton's method is used to solve the difference equations which include the asymptotic boundary conditions (4.9) on the image of $x=\tau$. Since this image is curved, the bandwidth of the Jacobian is increased and a bordering technique is used to solve for the Newton iterates. The computations were done on VAX 11/780 computers at the California Institute of Technology and at the Mathematics Research Center at the University of Wisconsin. 
In the first set of computations we present results for $a=0.463, d=0.4$, and $\kappa=3.51$. This is very close to the critical value $\kappa^{*}$ beyond which no real solutions of (4.5) exist. The minimum eigenvalue of the linearized problem about $u_{\infty}(y)$ in this case is $\lambda_{1}^{2}=0.66825$. Thus the decay to $u_{\infty}(y)$ as $x \rightarrow \infty$ is not very rapid. We have used each of the boundary conditions in (4.9) at each of four values of $\tau$ given in Table I. These $\tau$ values were determined by specifying the number of mesh intervals between the step and the artificial boundary to the right of the step to be $N=2$, $N=5, N=10$, and $N=20$, respectively. To get an accurate solution, to which we compared all the others, we used $\tau=2.262$ with the quadratic asymptotic boundary conditions; this solution is labeled $u^{h}$. The others are denoted by $u_{\mathrm{appx}}^{h}$ and in Table I we list the values of $\left\|u^{h}-u_{\text {appx }}^{h}\right\|_{\infty}$ for all twelve test computations. As expected, for each fixed $\tau$ value the quadratic approximation yields the best agreement, the zero-order case is worst, and the agreement improves as $\tau$ increases.

\section{TABLE I}

Comparison of "errors" in numerical solutions using different order asymptotic boundary conditions imposed at a variety of artificial boundaries. The "tail" is the area between the step and the artificial boundary (in the mapped domain).

\begin{tabular}{|c|c|c|c|c|}
\hline$\tau$ & $\begin{array}{l}N=\text { no. vert. mesh } \\
\text { lines in "tail" }\end{array}$ & $\begin{array}{l}\text { ABC appx. } \\
\quad \text { order }\end{array}$ & $\left\|u^{h}-u_{\text {appx. }}^{h}\right\|_{\infty}$ & $\max _{x=\tau}\left|u^{h}-u_{\infty}\right|$ \\
\hline .381 & 2 & $\begin{array}{l}0 \\
1 \\
2\end{array}$ & $\begin{array}{l}.2131 \\
.0213 \\
.0105\end{array}$ & .341 \\
\hline .520 & 5 & $\begin{array}{l}0 \\
1 \\
2\end{array}$ & $\begin{array}{l}.1936 \\
.0154 \\
.0051\end{array}$ & .269 \\
\hline .764 & 10 & $\begin{array}{l}0 \\
1 \\
2\end{array}$ & $\begin{array}{l}.1472 \\
.0082 \\
.0018\end{array}$ & .177 \\
\hline 1.262 & 20 & $\begin{array}{l}0 \\
1 \\
2\end{array}$ & $\begin{array}{l}.0743 \\
.0030 \\
.0015\end{array}$ & .076 \\
\hline
\end{tabular}

These results are displayed graphically in Figures 2, 3, 4, and 5 where the level curves of $u$ are approximated by interpolation from $u_{\text {appx }}^{h}$ for two different $\tau$ values and two different orders of ABC. In Figure 2 the solution is obtained on a "large" domain, $\tau=1.262$, using the quadratic ABC. In Figure 3 the same level curves are plotted from the solution obtained on a "small" domain, with $\tau=0.381$, using the quadratic $A B C$. These curves are superposed in Figure 4 and one can see that the errors decay as we move away from the artificial boundary toward the interior of the domain: $x<\tau$. This is as the theory and linear error analysis of [11], [12] predict. In Figure 5 we superpose the large domain solution of Figure 2 with the small domain solution obtained using the zero-order $\mathrm{ABC}: u_{x}(\tau, y)=0$. The level curves in the latter solution are quite distorted. 


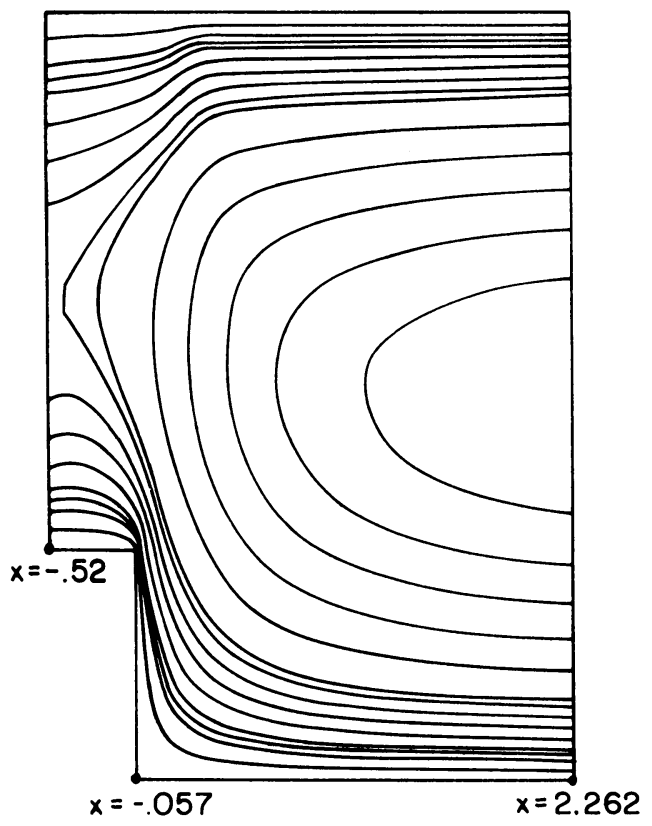

FigURE 2

Contour lines of $u=$ const. from the numerical solution using $\tau=1.262$ and the second-order $A B C$.

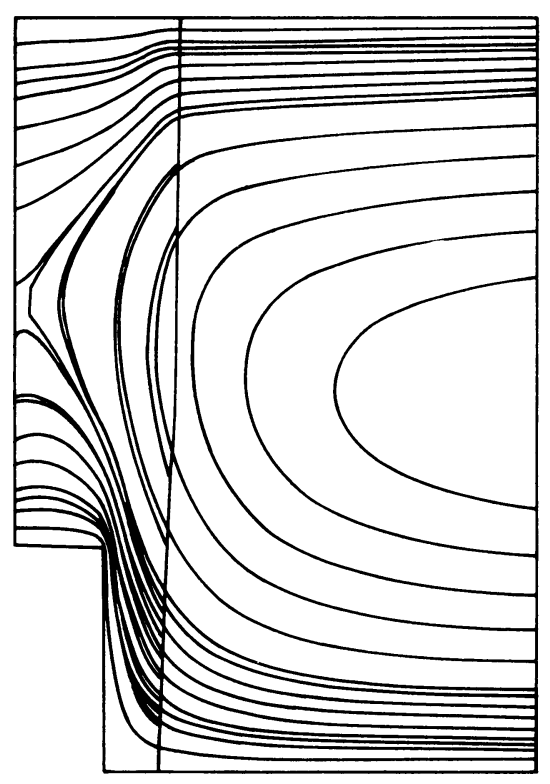

Figure 4

The contour lines of Figures 2 and 3 superposed.

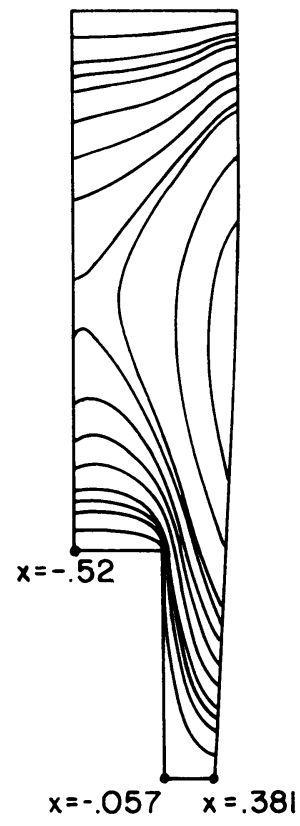

FIGURE 3

Same as in Figure 2 but with $\tau=0.381$.

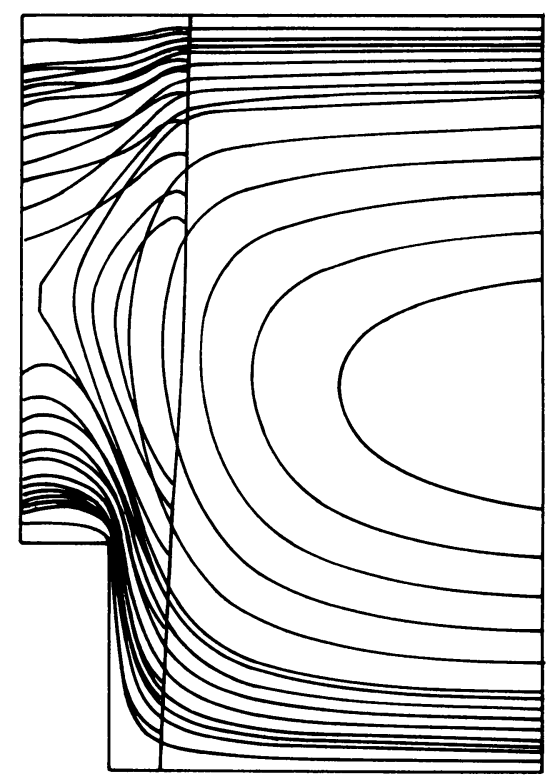

FIGURE 5

Contour lines of Figure 2 superposed with those from the numerical solution using $\tau=0.381$ and the zero-order $A B C$. 
Numerous other calculations were done with $d$ values in $[0,1]$, and the results were quite similar when $\kappa<\kappa^{*}$. This suggests that solutions of (4.1)-(4.4) exist for all $d \in[0,1]$ provided $\kappa \leqslant \kappa^{*}$. We do not know of existence theorems for such problems on infinite domains, although there is a large and ever-growing body of results for finite domain problems. Indeed, the early work of Keller and Cohen [16] treats the finite domain case for fairly general nonlinearities. Important extensions of this work are contained in Bandle [4] who proves that minimal positive solutions grow with the size of the domain. If these results can be extended to infinite domains, they suggest, in the present case, that for $d<0$ solutions may not exist for all $\kappa<\kappa^{*}$. That is, we conjecture that for fixed $a$ and $d<0$, there is a critical value

$$
\kappa^{* *}(a, d)<\kappa^{*}
$$

such that solutions of (4.1)-(4.4) exist for $\kappa<\kappa^{* *}(a, d)$, but do not exist for $\kappa>\kappa^{* *}(a, d)$.

In terms of the problem of reaction-diffusion or spontaneous combustion, our above conjecture implies that an area of excess thickness in the channel can cause ignition in a slab whose infinite extension is stable. This seems quite reasonable, physically, as an excess volume of reaction locally need not allow the transmission by diffusion to the thinner region of infinite extent where the excess heat is dissipated. If this balance cannot be accomplished sufficiently fast, then ignition could result, which implies no finite steady-state solution. So, indeed, this reasoning suggests that $\kappa^{* *}(a, d)$ is, for fixed $d>0$, a monotone decreasing function of $a$.

To get numerical evidence related to this conjecture we set $d=-0.4$ and considered a set of values of $a=x_{\text {step }}-x_{0}$, the half-length of the thicker part of the channel. Starting with small values of $\kappa \ll \kappa^{*}=3.51 \ldots$, for each particular $a$ value we use continuation in $\kappa$ to get good initial estimates for the solution at $\kappa+\Delta \kappa$. These are then used in Newton's method. As the number of iterations needed for convergence increases, we reduce $\Delta \kappa$, ultimately down to $\Delta \kappa=10^{-3}$. When Newton's method fails to converge in this sequence of continuations we have determined an estimate of $\kappa^{* *}(a, d)$. From the exact solution of the Bratu problem in a slab, (4.5)-(4.7), scaled to allow $0<y<L \equiv 1-d$ with $d<0$, we can easily compute $\kappa^{*}(d)$, the critical value for an infinite slab of thickness $L=1-d$. Clearly, in our above notation for the thickened stepped channel, we must have

$$
\kappa^{*}(d)=\kappa^{* *}(\infty, d) \text {. }
$$

From the scaled version of (4.7) we obtain, using (4.11), that

$$
\kappa^{* *}(\infty, 0)=3.5138 \ldots, \quad \kappa^{* *}(\infty,-.4)=1.791 \ldots
$$

Our continuation computations for the thickened slabs give us the following estimates:

$$
\begin{aligned}
& \text { (a) } \kappa^{* *}(0.7415,-.4) \doteq 2.049 \\
& \text { (b) } \kappa^{* *}(1.028,-.4) \doteq 1.978 \\
& \text { (c) } \kappa^{* *}(2.013,-.4) \doteq 1.858 \\
& \text { (d) } \kappa^{* *}(2.994,-.4) \doteq 1.821
\end{aligned}
$$

These results agree with the conjectured behavior of $\kappa^{* *}(a, d)$. Furthermore, since $\kappa^{* *}(0,-.4)=\kappa^{* *}(\infty, 0)$ for these problems, we see that the results lie in the interval determined by (4.12); that is, between the critical values for uniform slabs of the thickest or thinnest parts of the nonuniform slab. 


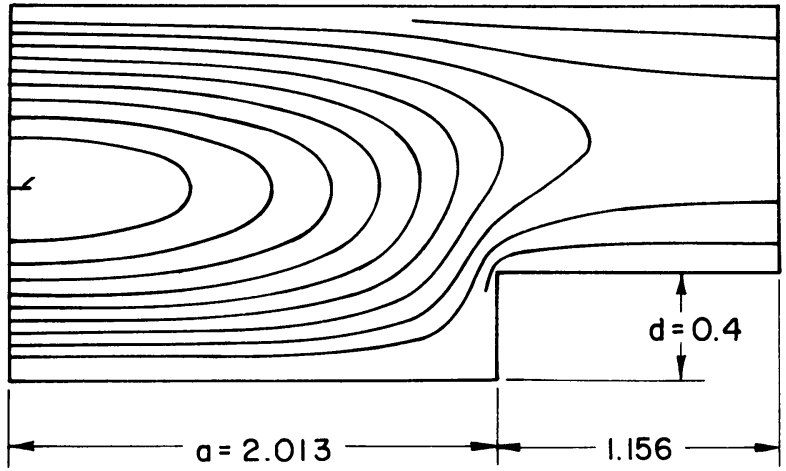

FIGURE 6

Contour lines of $u=$ const. from the numerical solution using $a=2.013$, $d=-0.4, \tau=1.247$ and $\kappa=1.857$. The critical $\kappa$ value in this configuration was estimated to be $\kappa_{\mathrm{cr}}=1.858$.

In Figure 6 we plot level curves of the solution for the cases $a=2.013, d=-0.4$, and $\kappa=1.857$. This is very close to the approximate critical value in (4.13(c)). The maximum value of $u$ is 1.27 , and it occurs at the left boundary. At $x \rightarrow \infty$ the maximum value is 0.3 .

Department of Applied Mathematics and Statistics

SUNY at Stony Brook

Stony Brook, New York 11794

Department of Applied Mathematics

California Institute of Technology

Pasadena, California 91125

1. S. Agmon, Lectures on Elliptic Boundary Value Problems, Van Nostrand, Princeton, N. J., 1965.

2. S. Agmon \& L. Nirenberg, "Properties of solutions of ordinary differential equations in Banach space," Comm. Pure Appl. Math., v. 16, 1963, pp. 121-239.

3. R. ARIS, The Mathematical Theory of Diffusion and Reaction in Permeable Catalysts, vol. I, Clarendon Press, Oxford, 1975.

4. C. BANDle, "Existence theorems, qualitative results and a priori bounds for a class of nonlinear Dirichlet problems," Arch. Rational Mech. Anal., v. 58, 1975, pp. 219-238.

5. A. Bayliss, C. Goldstein \& E. Turkel, "An iterative solution method for the Helmholtz equation." (To appear.)

6. J. BeREZANSKII, Expansions in Eigenfunctions of Selfadjoint Operators, Transl. Math. Monos., vol. 17, Amer. Math. Soc., Providence, R. I., 1968.

7. G. Fix \& S. MARIN, "Variational methods for underwater acoustic problems," J. Comput. Phys., v. 28,1978 , pp. 253-270.

8. I. Gohberg \& M. KREIN, Introduction to the Theory of Linear Nonselfadjoint Operators, Transl. Math. Monos., vol. 18, Amer. Math. Soc., Providence, R. I., 1969.

9. C. Goldstein, "A finite element method for solving Helmholtz type equations in waveguides and other unbounded domains," Math. Comp., v. 39, 1982, pp. 309-324.

10. B. GuSTAFsSON \& H.- O. Kreiss, "Boundary conditions for time dependent problems with an artificial boundary,” J. Comput Phys., v. 30, 1979, pp. 333-351.

11. T. Hagstrom \& H. B. Keller, "Exact boundary conditions at an artificial boundary for partial differential equations in cylinders," SIAM J. Math. Anal., v. 17, 1986, pp. 322-341.

12. T. Hagstrom, Reduction of Unbounded Domains to Bounded Domains for Partial Differential Equation Problems, Ph.D. Thesis, Applied Mathematics, California Institute of Technology, Pasadena, Calif., 1983.

13. A. Jepson, Asymptotic Boundary Conditions for Ordinary Differential Equations, Part I, Ph.D. Thesis, Applied Mathematics, California Institute of Technology, Pasadena, Calif., 1980. 
14. A. JePson \& H. B. Keller, "Boundary value problems on semi-infinite intervals. I. Linear problems," Numer. Math. (To appear.)

15. H. B. Keller, Numerical Solution of Two Point Boundary Value Problems, No. 24, CBMS/NSF Regional Conference Series on Applied Mathematics, SIAM, Philadelphia, Pa., 1976.

16. H. B. Keller \& D. S. COHEN, "Some positone problems suggested by nonlinear heat generation," J. Math. Mech., v. 16, 1967, pp. 1361-1376.

17. H. B. Keller \& M. Lentini, "Boundary value problems on semi-infinite intervals and their numerical solution,” SIAM J. Numer. Anal., v. 17, 1980, pp. 577-604.

18. H.- O. KREISS, "Difference approximations for boundary and eigenvalue problems for ordinary differential equations," Math. Comp., v. 26, 1972, pp. 605-624.

19. M. Lentini, Boundary Value Problems Over Semi-Infinite Intervals, Ph.D. Thesis, Applied Mathematics, California Institute of Technology, Pasadena, Calif., 1978. 